\title{
An Overview of Genetic Information of Latent Mycobacterium tuberculosis
}

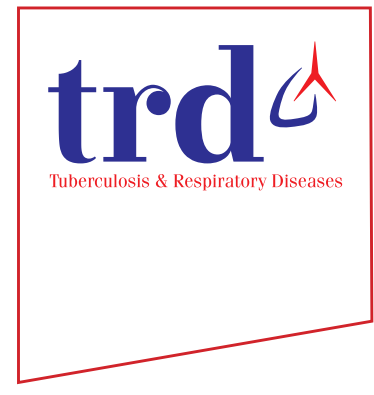

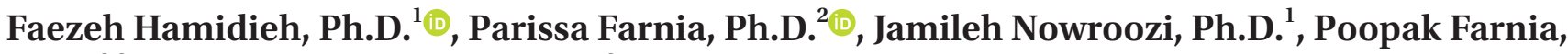 \\ Ph.D. ${ }^{2,3}$ and Ali Akbar Velayati, M.D. ${ }^{2}$ \\ ${ }^{1}$ Departement of Microbiology, Faculty of Biological Sciences, North Tehran Branch, Islamic Azad University, Tehran,
} ${ }^{2}$ Mycobacteriology Research (MRC), National Research Institute of Tuberculosis and Lung Disease (NRITLD), Shahid Beheshti University of Medical Sciences, Tehran, ${ }^{3}$ Department of Biotechnology, School of Advanced Technology in Medicine, Shahid Beheshti University of Medical Sciences, Tehran, Iran

Mycobacterium tuberculosis has infected more than two billion individuals worldwide, of whom 5\%-10\% have clinically active disease and $90 \%-95 \%$ remain in the latent stage with a reservoir of viable bacteria in the macrophages for extended periods of time. The tubercle bacilli at this stage are usually called dormant, non-viable, and/or non-culturable microorganisms. The patients with latent bacilli will not have clinical pictures and are not infectious. The infections in about $2 \%-23 \%$ of the patients with latent status become reactivated for various reasons such as cancer, human immunodeficiency virus infection, diabetes, and/or aging. Many studies have examined the mechanisms involved in the latent state of Mycobacterium and showed that latency modified the expression of many genes. Therefore, several mechanisms will change in this bacterium. Hence, this study aimed to briefly examine the genes involved in the latent state as well as the changes that are caused by Mycobacterium tuberculosis. The study also evaluated the relationship between the functions of these genes.

Keywords: Mycobacterium tuberculosis; Latency; Tuberculosis; Dormancy; Hypoxic Conditions

\section{Introduction}

Mycobacterium tuberculosis (MTB) which is at least as old as human life on Earth, is one of the major challenges to human health ${ }^{1,2}$. Infection with MTB has been estimated in approximately one third of the global population ${ }^{3}$. The World

Address for correspondence: Parissa Farnia, Ph.D.

Mycobacteriology Research (MRC), National Research Institute of

Tuberculosis and Lung Disease (NRITLD), Shahid Beheshti University of

Medical Sciences, Tehran 19569-44413, Iran

Phone: 98-21-27123000-26105050, Fax: 98-21-26109490

E-mail: pfarnia@hotmail.com

Received: Sep. 18, 2020

Revised: Oct. 1, 2020

Accepted: Oct. 30, 2020

Published online: Oct. 30, 2020

(c) It is identical to the Creative Commons Attribution Non-Commercial License (http://creativecommons.org/licenses/by-nc/4.0/).
Health Organization (WHO) reported that MTB is a preeminent cause of mortality (1.8 million deaths) worldwide in 2018, which is responsible for more deaths than human immunodeficiency virus and malaria ${ }^{4}$. Tuberculosis (TB)-infected persons are classified into two groups as (1) those with active TB presenting clinical or radiological manifestations of infection supported by laboratory evidence, (2) and those with latent tuberculosis infection (LTBI), which is an asymptomatic clinical presentation considering the largest reservoir for potential transmission ${ }^{5}$. Approximately $23 \%$ ( 1.7 billion people) of the world's overall population suffers from $\mathrm{LTBI}^{3}$.

The term LTBI was first proposed by Von Pirquet (1907), the 'Godfather' of the tuberculin skin test when he detected tuberculin skin reactions of $\geq 5 \mathrm{~mm}$ in children who did not manifest tuberculosis ${ }^{6}$. Lateral McCune et al. (1956-1996) showed the persistence of latent tubercle bacilli for extended times after chemotherapy, shortly after the launch of isoniazid. Their well-designed investigation, named the 'Cornell model' by the academic institute wherein the research was conducted, can be regarded a main work on mycobacterial latency and its relation to tuberculosis chemotherapy ${ }^{7}$. The abundant contingent indications from consideration of the natural history 
of tuberculosis in humans and experimental animals indicate that MTB is able to adapt to prolong periods of dormant status in tissues, and that such dormant bacilli are the causative agent for latency of the disease itself. Besides, the dormant bacilli can resist eradication by antimycobacterial agents ${ }^{8}$. Accordingly, the WHO end TB Strategy targets treatment of diseased people as well as LTBI cases who are at the risk of advancement to TB disease. Therefore, prevention of LTBI development to active TB seems to be an essential public health objective, which could markedly decline the TB reservoirs ${ }^{9}$.

The risk of TB recurrence during the life of an individual with confirmed LTBI is predicted to be $5 \%-15 \%$, and most of them develop active TB during the first 5 years following early infection ${ }^{10}$. Nonetheless, the probability of LTBI development to TB disease is dependent upon various factors including bacterial, host, and environmental parameters. Basically, LTBI can be remarked as an equilibrium condition between host and mycobacteria ${ }^{4}$. In response to MTB infection, most patients represent a robust immune response, resulting a low bacillary burden in the absence of clinical and radiographic findings. In this situation, the host immune response prevents developing active disease, and the tubercle bacilli evade immune elimination ${ }^{11}$.

In LTBI stage, bacilli can survive in a modified physiological state in MTB-infected macrophages and escape to activity immune system ${ }^{12}$. However, despite several years of examination, the accurate locality of the latent mycobacteria is still mysterious. Moreover, in some cases of LTBI, persistent bacilli appear as the antibiotic-tolerant organisms which are nonreplicating and exhibit reduced metabolic activity. Reduced susceptibility to elimination by cell wall structure inhibitor agents such as isoniazid is a general characteristic of persistent bacilli in $\mathrm{LTBI}^{13}$. The structural and physiological changes which occur permanently in MTB are associated with the expression of various genes, enabling the bacteria to survive under latency conditions $^{14}$. Therefore, for a better understanding of latency in MTB, the genes allowing bacteria to survive and escape from the immune system need to be more investigated ${ }^{15}$. In

Table 1. Genes involved in the latent stage

\begin{tabular}{|c|c|c|c|c|c|}
\hline Category & Identifier & Gene name & Product (probably) & $\begin{array}{l}\text { Length } \\
\text { (bp) }\end{array}$ & $\begin{array}{l}\text { Location } \\
\text { (bp) }\end{array}$ \\
\hline \multirow{6}{*}{$\begin{array}{l}\text { Genes involved in } \\
\text { metabolic changes }\end{array}$} & Rv0467 & icl & Isocitrate lyase & 1,287 & 557,527 \\
\hline & Rv1837c & $g l c B$ & Malate synthase g & 2,226 & $2,086,037$ \\
\hline & Rv1832 & $\operatorname{gcv} B$ & Glycine dehydrogenase & 2,826 & $2,075,877$ \\
\hline & Rv1161-4 & narGHJI & Nitrate reductase & 3,699 & $1,287,328$ \\
\hline & Rv1737c & narK2 & Putative nitrate/nitrite transporter NarK2 & 1,188 & $1,964,183$ \\
\hline & Rv1736c & $\operatorname{nar} X$ & Putative nitrate reductase NarX & 1,959 & $1,962,228$ \\
\hline \multirow{2}{*}{$\begin{array}{l}\text { Genes involved in cell } \\
\text { wall changes }\end{array}$} & Rv0470c & рсаA & Cyclopropane mycolic acid synthase 3 & 864 & 560,848 \\
\hline & Rv0126 & tres & Trehalose synthase & 1,806 & 152,354 \\
\hline \multirow[t]{13}{*}{ Signal transduction } & Rv3133c & $\operatorname{dos} R$ & Two-comp. resp. reg & 654 & $3,499,262$ \\
\hline & Rv3132c & $\operatorname{dos} S$ & Two-comp. resp. reg & 1,737 & $3,497,529$ \\
\hline & Rv2027c & $\operatorname{dos} T$ & Two-comp. resp. reg & 1,722 & $2,272,787$ \\
\hline & Rv0757 & phoP & $\begin{array}{l}\text { Possible two-component system response } \\
\text { transcriptional positive regulator PhoP }\end{array}$ & 744 & 851,608 \\
\hline & Rv0981 & mprA & $\begin{array}{l}\text { Two-component response transcriptional regulatory } \\
\text { protein MprA }\end{array}$ & 687 & $1,096,822$ \\
\hline & Rv1028c & $k d p D$ & Probable sensor protein KdpD & 2,583 & $1,149,104$ \\
\hline & Rv1027c & $k d p E$ & Probable transcriptional regulatory protein $\mathrm{KdpE}$ & 681 & $1,148,427$ \\
\hline & Rv3764c & $\operatorname{tcr} Y$ & Putative two component sensor kinase TcrY & 1,428 & $4,209,582$ \\
\hline & Rv1032c & $\operatorname{trcS}$ & Two-component sensor histidine kinase TrcS & 1,530 & $1,156,426$ \\
\hline & Rv3286c & $\operatorname{sig} F$ & AlteRNAtive RNA polymerase sigma factor SigF & 786 & $3,657,603$ \\
\hline & Rv3223c & $\operatorname{sig} H$ & RNA polymerase $\sigma$ factor & 651 & $3,598,901$ \\
\hline & Rv1221 & $\operatorname{sig} E$ & AlteRNAtive RNA polymerase sigma factor SigE & 774 & $1,364,413$ \\
\hline & Rv3416 & whiB3 & Transcriptional regulatory protein whib-like WhiB3 & 309 & $3,832,240$ \\
\hline
\end{tabular}


this regard, we tried to overview the genetic information of latency in $\mathrm{MTB}^{16}$. The general characteristics of the genes examined in this review are summarized in Table 1 . The genes investigated in this study are evaluated based on their functional relevance into three groups of (1) genes involved in metabolic changes, (2) genes involved in cell wall changes, and (3) signal transduction which are discussed below (Table 1).

\section{Genes Involved in Metabolic Changes in the Latency Stage}

One of the abilities of MTB strain is its quick adaptability with the environment ${ }^{17}$. A considerable number of studies demonstrate that metabolic pathways in MTB changed after entering the macrophage ${ }^{18}$. The presence of MTB in macrophages leads to granuloma formation in human's lung. The presence and survival of MTB in lung granulomas has been investigated in several studies, and it has been shown that MTB is likely to be present in the environment with conditions such as oxygen depletion, redox stress, increased carbon dioxide, nutrient degradation, and $\mathrm{pH}$ reduction ${ }^{19}$. In aerobic conditions of the MTB, the oxidative phosphorylation tricarboxylic acid (TCA) cycle is used to generate energy from the pathway of the electron transfer channel (ETC) for the proton transfer energy. In anaerobic conditions, which reduce oxygen levels, the bacteria will not be able to use conventional pathways to generate energy and use the ETC pathway to transmit electrons ${ }^{20,21}$. The adaptation of MTB to this condition requires the expression of different genes, in order to activate the appropriate metabolic pathways to supply the energy it needs. Modifying the metabolic process requires the alteration of the produced enzymes. The production of enzymes required for changes in the metabolic process necessitates altering the expression of genes involved in the production of these enzymes. In this section, the expression of $i c l, g l c \mathrm{~B}, g c v \mathrm{~B}$, narGHJI, narK2, and narX genes in latency and their effect on mycobacterial viability are investigated ${ }^{20,21}$. In Figure 1, it has been attempted to illustrate the modification of metabolic pathways and thus the alteration of production of the required enzymes in the metabolic cycles, which are caused by the expression of these genes.

\section{1.icl gene}

The icl gene MTB encodes the isocitrate lyase (ICL) ${ }^{22}$, which is the primary enzyme in the glyoxylate (GLX) cycle $^{23}$.icl gene characteristics of the gene are summarized in Table 1. This enzyme is necessary for the use of fatty acid by bacteria ${ }^{24}$ and important for latency in MTB, which is called "persistence factor $^{\prime 25}$. In 1998s, the icl gene was sequenced for the first time by Cole et $\mathrm{al}^{22}$ and has been studied extensively since then. This results in the change of isocitrate to GLX and succinate. When the MTB $\Delta i c l$ mutant was examined, it was shown that its activity had not changed in macrophage resting mode, while it had significantly reduced in active mode ${ }^{25}$. The main source of carbon and energy in MTB is fatty acids, which is provided by beta-oxidation. At the time of the hypoxic, ICL production increases significantly (five times). When the carbon source is reduced, the ICL enables the bacteria to use fatty acids as a source of carbon and energy using GLX ${ }^{26}$. Several studies have shown that the expression of the icl gene for MTB transmission is necessary from critical to stable status ${ }^{18}$ (Figure 1). The presence of this gene preserves MTB life in latency conditions and therefore contributes to the persistence of infection in the human's body ${ }^{27}$. Not only is the ICL a key enzyme in the survival of mycobacteria, but it also is not present in humans, so it can be considered a therapeutic target.

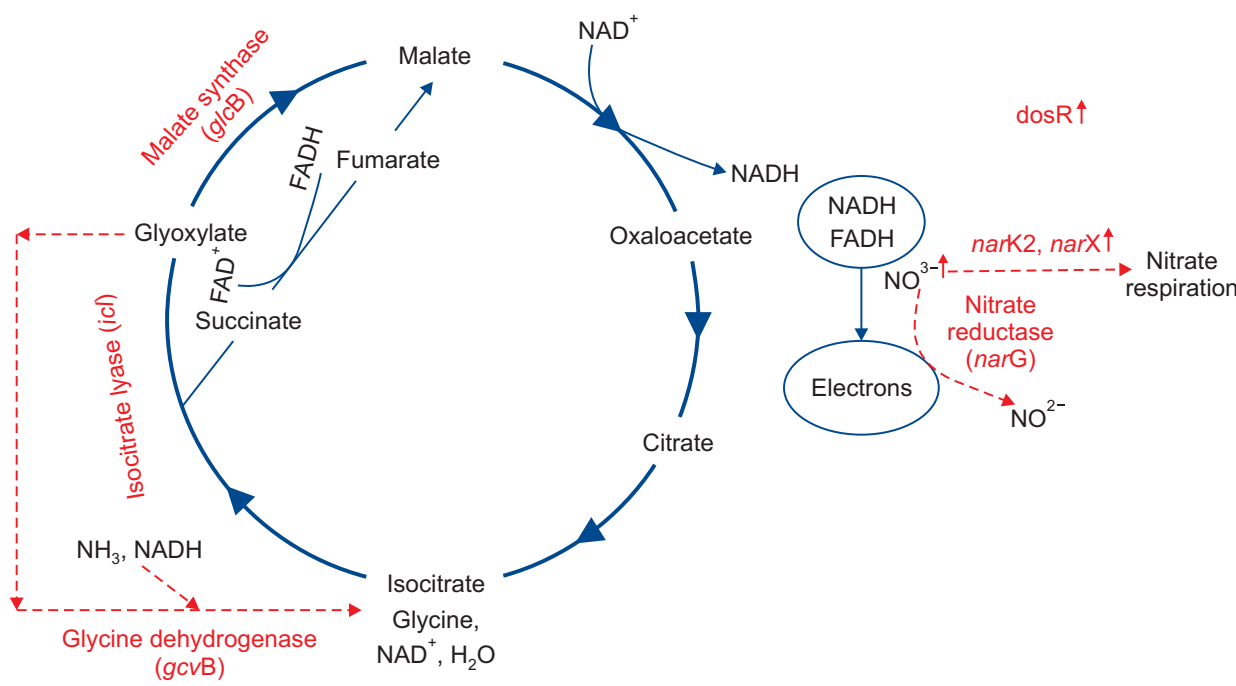

Figure 1. Metabolic changes induced by hypoxia. Enhanced expression of the icl gene caused the conversion of isocitrate to glyoxylate and succinate. The $g l c \mathrm{~B}$ and gcv B genes are involved in the conversion of glyoxylate to malate and glyoxylate to glycine, respectively. The electrons generated in this pathway may lead to nitrate reduction, which is also associated with increased dosR expression. Increased dos R expression also affects the expression of other genes such as narK2 and narX. 


\section{2.glcB gene}

Many studies have been done on $g l c B$ gene since it was the first identified ${ }^{28}$. The $g l c B$ gene codes the malate synthase, which leads to bypass of GLX, together with the production of the ICL enzyme by the $i c l$ gene $^{29}$. This probably enables the MTB pathway to adapt itself to anaerobic conditions and nutrient deficiencies. These two enzymes also provide the exchange of isocitrate to malate, which helps to keep continuing the TCA cycle $^{30}$. However, studies have shown that there was no significant increase in the production of this enzyme under hypoxic conditions ${ }^{29}$ (Figure 2). Since this enzyme is involved in the GLX, and this anaplerotic pathway is not present in mammals, it can be investigated as an option for treatment of tuberculosis.

\section{3.gcvB gene}

gcvB gene codes glycine dehydrogenase (GDH), which most likely encodes the $P$ protein of the glycine cleavage system (GCS) ${ }^{31}$. The function of this enzyme, first identified in MTB in 1962, was detected in nonreplicating persistent (NRP) $\mathrm{MTBs}^{32}$. As $\operatorname{gcv}_{\mathrm{C}}$ and $\operatorname{gcvH}_{\mathrm{C}}$ are located in the operon associated with glycine catabolism, the GCS may use glycine as a source of nitrogen ${ }^{33}$. Increasing the expression of this enzyme in hypoxia conditions in non-proliferative MTB is shown ${ }^{8}$. The GDH catalyzes the amination of glyoxalate to glycine and simultaneously catalyzes the oxidation of NADH to NAD ${ }^{8}$.
The activity of GDH has been shown to be stable in vitro ${ }^{32}$. Although it showed that the $g_{c v} B$ gene is involved in the expression of the GDH enzyme, other genes are also involved in this process, and further study of the interaction of these genes on this enzyme is needed.

\section{4. narGHJI gene}

This gene codes nitrate reductase ${ }^{34}$. Although nitrate reductase enzyme activity encoded by this gene is low in aerobic conditions, it increases in microaerophile ${ }^{35}$. Such a function may be related to the compliance of MTB in a permanent condition $^{36}$. However, it has been shown that hypoxic conditions do not affect narGHJI expression ${ }^{36}$. It is noteworthy that even though nitrate reductase is unaffected, when oxygen is gradually depleted (over a period of more than 46 days), nitrate release reductase increases in the event of an immediate discharge of oxygen ${ }^{36}$. Nitrate is reduced to nitrite by nitrate reductase to produce bacterial energy to enter the NRP step ${ }^{29}$. It has been shown that the activity of nitrate reductase in MTB leads to the continuation of the electron transfer chain under microaerophilic or anaerobic conditions ${ }^{37}$. In addition to the narGHJI gene, the narK2 and narX genes are also involved in nitrate reduction, which are discussed below.

\section{5. narK2 gene}

nark2 gene encodes the transporter nitrate, which is ex-

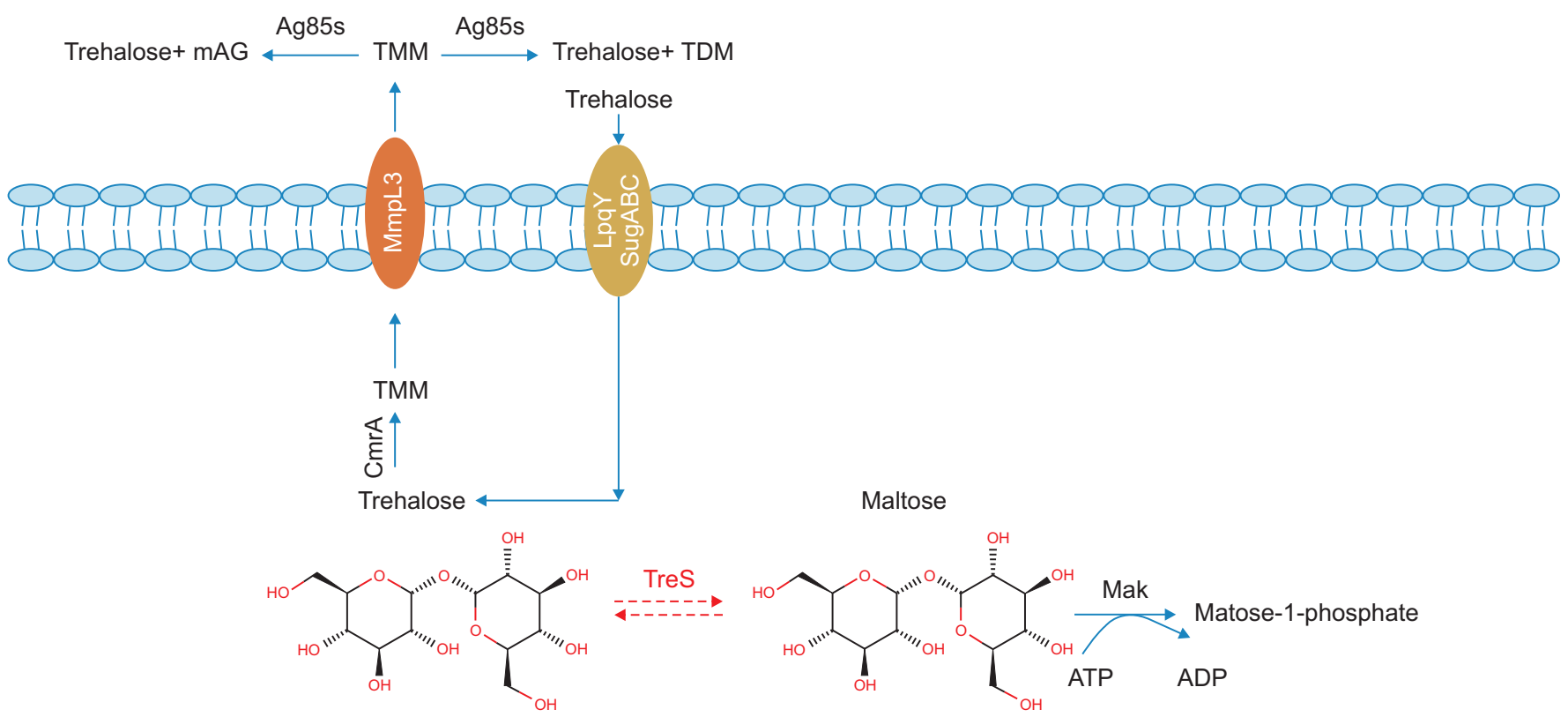

Figure 2. TreS enzyme functions in the Mycobacterium tuberculosis cell wall structure. This enzyme catalyzes the reversible interconversion of maltose and trehalose. Maltose is converted to maltose-1 phosphate by Mak and trehalose is converted to trehalose monomycolate (TMM) by CmrA, which crosses the cell membrane via MmpL3. Antigen 85 (Ag85s) complex enzymes catalyze the conversion of TMM to trehalose dimycolate (TDM), which plays a significant role in protecting cells under stress. 
pressed in hypoxic conditions ${ }^{38}$. The release of nark2 in hypoxia conditions probably increases nitrite production in $\mathrm{MTB}^{39}$. Unlike narGHJI, it has been shown that narK2 is produced in response to the presence of nitrate or nitrite $e^{40}$. Another contrast with narGHJI is that, unlike narGHJI, the expression of narK2 increases in hypoxia and decreases in nitric oxide levels ${ }^{41}$. This increase in activity is probably due to the fact that the hypoxia and the presence of NO lead to the inactivation of cytochrome oxidase and the activation of $\operatorname{narK} 2^{42}$. It has been shown that $\triangle$ narK2 mutant does not affect the expression of hypoxia ${ }^{41}$. The NarK2 protein is positioned on the surface of the membrane, and can detect the oxygen level of the environment as a sensor, which has been shown to maintain the amount of ATP in hypoxia conditions ${ }^{40}$. The DosR/ DevR controller transcribes narK2 in hypoxic situations and reduces $\mathrm{NO}$ levels ${ }^{43}$.

\section{6. narX gene}

nar $X$ gene encodes fused nitrate reductase ${ }^{31}$. The studies have shown that $\operatorname{nar} X$ is homologous with nitrate reductase proteins in other prokaryotes ${ }^{31}$. Although the function of narX has not been completely detected, studies have shown that the expression of this gene in the presence of NO increases over 800 times $^{44}$. NarX is a membrane protein which is likely to contribute to the electron transport chain ${ }^{45}$. It has been suggested that in the absence of oxygen, NarX in the environment can lead to respiratory depression of nitrate as an electron transfer ${ }^{44}$. It has been determined that $\operatorname{nar} X$ is the first gene to be replicated in latent MTB ${ }^{45}$. Therefore, it is suggested that the expression of this gene be used as a marker for latent MTB diagnosis ${ }^{44}$. However, nark2 is expected for induction of nitrates reductase activity in anaerobic conditions, but narX is not required ${ }^{44}$. Nitrate reduction is one of the activities performed under hypoxia at the latency stage, and the investigation of the genes involved in this activity is important to better understand the latency process.

\section{Genes Involved in Cell Wall Changes in the Latency Stage}

One of the characteristics of MTB is the unique cellular wall, due to which the bacterium inside the cell survives. The significant part of this structure are the lipids, and mycolic acid consists of $60 \%$ of the cell wall lipids. In the cell wall, there are high molecular weight fatty acids (60-90 carbon), which are common to all mycobacteria species ${ }^{46}$. One of the unique characteristics of MTB cell cover is the presence of 2-alkyl, mycolic acid, and 3-hydroxy fatty acids. The cellular structure of MTB prevents the immune system response to the bacteria. Furthermore, MTB cell wall compounds make it flexible and fluid. The formation of granuloma is one of the reasons for
MTB survival, so MTB is stationary in the latent state of granuloma. One of the reasons for the formation of granuloma is the presence of the structure of trehalose-6,6'-dimycolate (TDM), which is also known as the cord-factor ${ }^{47}$. TDM is identified by a receptor called the minacle located on the macrophage, which creates granuloma with NO production ${ }^{48}$. Another effect of this lipid is its inhibitory effect on the migration of leukocytes, which plays a significant function in the survival of the bacteria. In addition to TDM, latent changes occur in the MTB cell wall, which is associated with a change in the expression of the genes related to it $^{49}$. These genes are briefly discussed in the following sections.

\section{1.pcaA}

The $p c a A$ gene codes the cyclopropane synthase enzyme ${ }^{50}$. The activity of this enzyme is required for cyclopropanation of mycolic acid ${ }^{51}$. PcaA phosphorylation leads to a decrease in the synthesis of cyclopropane, involving the exchange of mycolic acid profiles ${ }^{52}$. One of the effects of changing the mycolic characterization profile is the stoppage of intracellular bacterial replication and the absence of formation of a phagosome maturation block (PMB $)^{52}$. The $\triangle p c a A$ mutant study has revealed that this mutant does not have the ability to produce any serpentine cords and not only does not lead to persistence in mouse models but also results in the death of the mous $\mathrm{e}^{52}$. As mentioned before, PcaA is implicated in the formation of PMB and mycolic acid, which leads to the survival of MTB in the host macrophages in latent stage ${ }^{51}$. The results of the studies indicate that pcaA gene activity is required for mycobacterial cell survival and is needed for the escape of macrophage killing.

\section{2. treS}

treS gene encodes the trehalose synthase (TreS) enzyme ${ }^{53}$. TreS substrates are trehalose or maltose, but the ability to produce trehalose from maltose is 2.5 times $^{54}$. Trehalose is located in the cytoplasm and glycolipids cell wall of mycobacterium $^{55}$. This compound not only acts as a virulence factor, but also has many other roles ${ }^{54}$. Among different roles of trehalase is the one that it can be used as carbon and energy source as well as its inhibitory effect on bacterial drying and freezing ${ }^{56}$. Although treS removal has no effect on the amount of intracellular trehalose, its expression increases the amount of trehalose in the cell, which indicates the role of this gene in the concentration of intracellular maltose $e^{54}$. One of the structures of trehalose is TDM, which is one of the important pathogenicity factors (Figure 2$)^{54}$. As previously stated, TDM plays a pivotal role in the survival of MTB by formation of granuloma and inhibiting the formation of phagosome lysosome fusion ${ }^{54}$. Since trehalose plays both a major role in the cell wall structure and protects mycobacteria under environmental stress condi- 
tions, investigating the genes involved in trehalose utilization pathways might be useful for better understanding the latency stage.

\section{Signal Transduction and Latency}

Signal transduction is one of the factors affecting MTB survival in a granuloma, which contributes to bacterial survival under conditions such as acidity decrease, nutrient deficiencies, and environmental oxygen depletion ${ }^{57}$. In this review, two-component systems (TCS) and sigma factors are examined in detail.

\section{Two-component systems}

Eleven TCS have been identified in MTB, which is far lower than other bacteria, including Escherichia coli (more than $30)^{58}$. TCS in Mycobacterium has several physiological functions, but these functions are not completely understood ${ }^{59}$. One of the functions of TCS is to help maintain bacterial living under environmental degradation conditions (such as macrophage environment or antimicrobial agents), and TCS is an extremely effective factor in bacterial pathogenesis ${ }^{60}$. The TCS includes sensor histidine kinase (HK), which, using phosphorylation cognate response regulator $(\mathrm{RR})$, regulates the expression of the gene and creates an appropriate response to the sensor ${ }^{59}$. A significant characteristic of TCS, its presence in all prokaryotes and its nonbeing in high-level eukaryotes, has made it a valuable treatment option ${ }^{61}$. It has been shown that the amount of pathogenicity in mutant lacking TCS considerably decreases ${ }^{58}$. TcrX and TcrY of open reading frames play an important role in coding RR and HK, respectively ${ }^{62}$.

\section{1) dosR}

The dosR regolone consists of approximately 50 genes (Table 2) ) $^{63,64}$, which are essential for survival in the latent pe$\operatorname{riod}^{65}$. DosR is a transcritical factor, which plays a significant role in adapting to the initial conditions of hypoxia, reducing $\mathrm{NO}$ and carbon monoxide levels ${ }^{66}$. It has been revealed that DosR leads to the regulation of the TAG (TAG production gene tgs 1) gene, Tgs (TAG), which also stops the production of TAG by removing DosR, and the increase of TAG production by provoking DosR ${ }^{67}$. The DosR regolon promotes the production of antigens, which are identified by T-cells at latency, and are essential for controlling infection. It is likely that T-cell response is due to the induction of interleukin (IL)-10, IL-17, and interferon- $\gamma$ production in the latent condition ${ }^{68}$.

In this two-compound system, DosS and DosT act as HK, which activate DosR ${ }^{69}$. Transcription of the dosR, dosS genes occur together, possibly due to the genetic association of them ${ }^{70}$. The dosR and dos $S$ genes are considered preserved genes, while the $\operatorname{dos} T$ gene is preserved less ${ }^{71}$. Studies have
Table 2. Genes regulated by dosR under hypoxic conditions

\begin{tabular}{|c|c|c|}
\hline Gene name & Identifier & Function \\
\hline Rv0079 & Rv0079 & Unknown \\
\hline Rv0080 & Rv0080 & Unknown \\
\hline Rv0081 & Rv0081 & $\begin{array}{l}\text { Involved in transcriptional } \\
\text { mechanism }\end{array}$ \\
\hline Rv0082 & Rv0082 & Unknown \\
\hline Rv0083 & Rv0083 & Unknown \\
\hline Rv0569 & Rv0569 & Unknown \\
\hline$n r d Z$ & Rv0570 & $\begin{array}{l}\text { Involved in the DNA replication } \\
\text { pathway }\end{array}$ \\
\hline Rv0571c & Rv0571c & Unknown \\
\hline Rv0572c & Rv0572c & Unknown \\
\hline Rv0574c & Rv0574c & Unknown \\
\hline МТ0639 & MT0639 & Unknown \\
\hline Rv1733c & Rv1733c & Unknown \\
\hline Rv1734c & Rv1734c & Unknown \\
\hline $\operatorname{narX}$ & Rv1736c & Involved in nitrate reduction \\
\hline narK2 & Rv1737c & Involved in excretion of nitrite \\
\hline Rv1738 & Rv1738 & Unknown \\
\hline Rv1812c & Rv1812c & Unknown \\
\hline Rv1813c & Rv1813c & Unknown \\
\hline Rv1996 & Rv1996 & Unknown \\
\hline $\operatorname{ctpF}$ & Rv1997 & Metal cation-transporting ATPase \\
\hline Rv2003c & Rv2003c & Unknown \\
\hline Rv2004c & Rv2004c & Unknown \\
\hline Rv2005c & Rv2005c & Unknown \\
\hline otsB1 & Rv2006 & Involved in trehalose biosynthesis \\
\hline$f d x \mathrm{~A}$ & Rv2007c & Involved in electron transfer \\
\hline Rv2028c & Rv2028c & Unknown \\
\hline$p f k \mathrm{~B}$ & Rv2029c & Involved in glycolysis \\
\hline Rv2030c & Rv2030c & Unknown \\
\hline hspX & Rv2031c & Stress protein induced by anoxia \\
\hline $\operatorname{acg}$ & Rv2032 & Unknown \\
\hline TB31.7 & Rv2623 & Unknown \\
\hline Rv2624c & Rv2624c & Unknown \\
\hline Rv2625c & Rv2625c & Unknown \\
\hline hrp 1 & Rv2626c & Unknown \\
\hline Rv2627c & Rv2627c & Unknown \\
\hline Rv2628 & Rv2628 & Unknown \\
\hline Rv2629 & Rv2629 & Unknown \\
\hline Rv2630 & Rv2630 & Unknown \\
\hline Rv2631 & Rv2631 & Unknown \\
\hline vapB22 & Rv2830c & Unknown \\
\hline
\end{tabular}


Table 2. Continued

\begin{tabular}{|c|c|c|}
\hline Gene name & Identifier & Function \\
\hline Rv3126c & Rv3126c & Unknown \\
\hline Rv3127 & Rv3127 & Unknown \\
\hline $\operatorname{Rv} 3128 c$ & Rv3128c & Unknown \\
\hline Rv3129 & Rv3129 & Unknown \\
\hline $\operatorname{tgs} 1$ & Rv3130c & $\begin{array}{l}\text { Involved in synthesis of } \\
\text { triacylglycerol }\end{array}$ \\
\hline Rv3131 & Rv3131 & Unknown \\
\hline $\operatorname{dev} S$ & Rv3132c & $\begin{array}{l}\text { Sensor part of the two-component } \\
\text { regulatory system DEVR/DEVS/ } \\
\text { dost }\end{array}$ \\
\hline $\operatorname{dev} R$ & Rv3133c & $\begin{array}{l}\text { Regulator part of the two- } \\
\text { component regulatory system } \\
\text { DEVR/DEVS/dost }\end{array}$ \\
\hline Rv3134c & Rv3134c & Unknown \\
\hline bfr $B$ & Rv3841 & Involved in iron storage \\
\hline
\end{tabular}

shown that DosR activity is necessary for the adaptation of MTB in hypoxia conditions ${ }^{69}$. It is also observed in studies that, despite the structural similarities in DosT and DosS, these proteins are functionally different, so DosT acting as a direct oxygen sensor and DosS as a redox sensor ${ }^{66}$. Since the expression of $d o s R$ regolon increases in the latency stage, and $\mathrm{T}$ cells show specific responses to some of the antigens which are specific to the regolon, it is considered a candidate for the production of a vaccine and drug.

\section{2) phoP gene}

The $p h o P$ gene codes the transcriptional regulator of the TCS PhoPR. PhoP has been distinguished as having several functions that appear to be essential during latency to keep the bacteria alive ${ }^{72}$. phoP leads to the regulation of the expression of the genes of $p k s 2$ (induction of sulfolipids production), pks3 (induction of polyacyltrehalose/diacyltrehalose production) and ald (induction of L-alanine dehydrogenase production $)^{73}$. This $p h o P$ also provides the metabolism of cell wall fats, the regulation of the intestinal acidity of the cell, the adaptation to the conditions of thermic stress, and the response to the early stages of hypoxia in $\mathrm{MTB}^{74}$. Another significant point is that of the expression of the icl gene is negatively regulated by $p h o P$. It has been designated that although $\triangle p h o P$ mutant does not have the capability to replicate, it can survive in the macrophage ${ }^{72}$. Although the phoP gene is required for mycobacterial intracellular growth, the need for it in the latency is still controversial.

\section{3) $\mathrm{mprA}$}

The mprA gene encodes the two-component regulator ${ }^{31}$. MprA is part of the MtrA-MtrB system, which was the first
TCS system to be distinguished in the $1990 \mathrm{~s}^{75}$. This system is involved in controlling MTB replication in macrophages and regulating significant processes of MTB physiology ${ }^{57,76}$. Studies have shown that the expression of the mprA gene is effective in maintaining latency infection in MTB so that the $\Delta m p r A$ mutant does not have the ability to keep the infection in a latent status ${ }^{77}$. The role of MprA in regulating the expression of the DosR regolum and Rv1813, which is involved in regulating the expression of type II NADH dehydrogenase, is shown in some unfavorable environmental conditions ${ }^{78}$. Identification of regulatory functions of MprA leads to a better understanding of the mechanisms involved in latency stage in the host and will ultimately lead to a better understanding of how to establish TB control in the latency stage.

\section{4) kdpDE}

The two-component kdpDE system is expressed by the $R v 1027 c$ and $R v 1028 c$ genes $^{79}$. It has been reported that kdpDE TCS is present in many species of bacteria (more than 1,000 species), which are extensively protected ${ }^{80}$. The expression of $k d p D E$ increases in incompatible conditions such as reduced nutrients, temperature stress, acidity, and hypoxia. In vivo, $k d p D$ expression increases in nutrient depravity conditions, and expression of $k d p E$ also increases during growth in macrophages ${ }^{81}$. Both $k d p D$ and $k d p E$ genes are located on an operon, and they are expressed in a particular variant. The position of phosphorylation in KdpD and KdpE is His642 and Asp52, respectively, where the phosphorylation of KdpD and the transfer of the phosphoryl group to KdpE leads to the response to fluctuations in the acidity of the environment ${ }^{79}$. The results of our studies indicate that kdpDE leads to a decrease in mycobacterial growth within the macrophage, which may be due to a change in the mycobacterial cell wall. This function can be important in preserving mycobacteria in the latency phase.

\section{5) tcrY}

The tcrY gene encodes the two-component regulator ${ }^{31}$.tcr $Y$ and $\operatorname{tcr} X$ are implicated in the formation of TCS TcrX/Y in $\mathrm{MTB}^{82}$. This system is protected in all species of Mycobacterium except Mycobacterium leprae ${ }^{62}$. It has been confirmed that in mouse models where tcrX/Y is eliminated, the severity of the disease increases, and the mouse model dies earlier ${ }^{83}$. TcrY contains three domains, including the C-terminal located in the outer the cell, a membrane domain, and the N-terminal part positioned in the space of cytosol ${ }^{82}$. TcrX is also generally located in the cytosol section ${ }^{84}$. TcrY is phosphorylated in the presence of $\mathrm{Mg}^{2+}$ or $\mathrm{Ca}^{2+}$ ions, which passes this phosphorus to $\mathrm{Tcr} \mathrm{X}^{84}$. It is shown that $t c r Y$ and $t c r X$ are apparently translated in one pathway ${ }^{84}$. Although the results of the present study indicate that tcrY is expressed under conditions of iron restriction and post-infection, little is known about the conditions affecting the expression of this two-component regulator; 
therefore, further studies are needed to investigate the effect of this gene on latency.

\section{6) $\operatorname{trcSR}$}

The trcSR TCS is coded by trcS and trcR, respectively ${ }^{85}$. In this TCS, TrcS with phosphorylation in the presence of $\mathrm{Mg}^{2+}$ or $\mathrm{Ca}^{2+}$ ions leads to the transfer of phosphorus to $\operatorname{TrcR}^{86}$. Despite studies of the function of this system, the only evidence that indicates the expression of TCS trcSR in latency is that the system is expressed at an early stage of infection in macrophages and in conditions of reduced oxygen in the environment ${ }^{84}$. However, its precise function is still not completely defined ${ }^{86}$.

\section{Sigma factors}

Sigma factors are present in all bacteria except in $\mathrm{Myco}$ plasma. They also regulate expression, therefore responding to environmental stress requires the formation of a network of factors so that the bacteria can survive. In addition, the reprogramming of RNA polymerase is required to translate the factor $\sigma^{87}$. In general, sigma factors are divided into two main groups, which include $\sigma 54$ and $\sigma 70^{88}$. In MTB, there is 1 (sigma) of the 'housekeeping' factor and $12 \sigma$ ancillary factors. Housekeeping $\sigma$ factor is expressed in response to natural conditions, while $\sigma$ ancillary factors are expressed in response to environmental stresses ${ }^{89}$. All of the $\sigma$ factors available in the MTB are $\sigma 70$, and the presence of this type of $\sigma$ factor has been confirmed in all bacterial species ${ }^{88,90}$.

\section{1) $\operatorname{sig} F$}

SigF expression occurs at the stationary stage as well as in the presence of environmental stress such as cold stress, nutrient depletion, acidity change, and the presence of drugs, particularly metronidazole ${ }^{89}$. SigF expression is regulated by Usf $\mathrm{x}^{91}$. In addition to being directly involved in the expression of 14 other genes, SigF is also engaged in the expression of other sigma factors. For example, it influences on viral virulence by affecting the expression of $\mathrm{SigC}^{92}$. Identification of genes involved in preserving bacterial life at the late stage of growth important to eliminate MTB at this stage because it can help identify the therapeutic process.

\section{2) $\operatorname{sigH}$}

Sigma $\mathrm{H}$ is expressed in response to high-temperature stress, oxidative thiol, and macrophage entry ${ }^{22,93-95}$. sigH gene, like most of the genes examined in this study, was identified by the MTB genes sequence in the $1980 \mathrm{~s}^{22}$. SigH is implicated in the translation of 31 structural genes, leading to the induction of sigB and $\operatorname{sigE}^{96}$. It is acknowledged that sigH is expressed when MTB enters the host cell, so the expression of this factor may be effective in provoking latency ${ }^{97}$. Thus, many studies have determined that $\Delta-\sigma H$ mutant in the murine model will not be able to induce normal granuloma ${ }^{95}$. sigH gene studies indicate that it plays an important role in the regulation of heat and oxidative stress response and is probably important in the pathogenesis of MTB.

\section{3) sigE}

SigE is involved in the expression of genes that lead to pathogenicity which has been studied in many studies ${ }^{95}$. SigE is expressed in response to oxidative stress, sodium dodecyl sulfate detergent, and high temperature, so the viability of sigE mutants would be much lower under these conditions, and its growth decreases in the granuloma and mouse mode ${ }^{95}$. Studies have also confirmed that the induced immune response to the sigE mutant would be more effective in the BALB/c mouse model, making this sigma a candidate for the vaccine ${ }^{95,98}$. Therefore, as a therapeutic option, further understanding of this gene's function and its implications seems to be a necessity.

\section{4) whiB}

WhiB3, a cytoplasmic redox sensor, is required to induce MTB resistance under acidic stress conditions ${ }^{99}$. WhiB3 regulates the expression of genes engaged in responding to decreased acidity, hypoxia, NO reduction, redox metabolism, and lipid anabolism ${ }^{100}$. It has been revealed that the MTB $\triangle$ whiB3 mutant greatly reduces its virulence severity in the mouse model. WhiB expression is induced during the early stage of rat lung infection and resting macrophages, but is suppressed in activated macrophages ${ }^{99}$. Although the function of whiB has been demonstrated in situations of changes in oxygen concentration, its precise role in MTB adaptation to these changes has not been elucidated, so further investigations in this area are essential.

\section{Conclusion}

"Latency", "persistence", and "dormancy" are common terms which are used interchangeably in related studies. When a person is infected by MTB, the bacterium is capable of persisting during a prolonged period in a process named LTBI. Conventionally, LTBI has been known to engage in the bacilli lasting in a non-duplicating state in old lesions but still maintaining their capability to cause reactivation and active TB upon occurrence of an immune response disturbance.

In this study, we attempted to induce the distinguished latency genes in MTB. Understanding the latency requires the identification of genes that can sustain MTB to survive in hypoxia, nutrient degradation, environmental stress, and acidity variations. Distinguishing these genes allows researchers to be able to study treatment options and new vaccines more precisely. Although many studies have been done to identify these genes, there are still many uncertainties about this issue. Therefore, further studies in this field are required in the labo- 
ratory environment and animal models to shed more light on the latency process.

\section{Authors' Contributions}

Conceptualization: Hamidieh F, Farnia P, Nowroozi J, Farnia P, Velayati AA. Validation: Farnia P, Velayati AA. Investigation: Farnia P, Hamidieh F. Writing - original draft preparation: Hamidieh F. Writing - review and editing: Farnia P, Nowroozi J, Farnia P. Approval of final manuscript: all authors.

\section{Conflicts of Interest}

No potential conflict of interest relevant to this article was reported.

\section{Acknowledgments}

This article is sponsored by Dr. Masih Daneshvari Hospital, Mycobacteriology Research Center (MRC), National Research Institute of Tuberculosis and Lung Disease (NRITLD) and they are thanked.

\section{Funding}

The project approved by scientific committee and funded by National Research Institute of Tuberculosis and Lung Disease (NRITLD), IR.SBMU.NRITLD.REC.1397.566.

\section{References}

1. Velayati AA, Farnia P, Masjedi MR. Latent tuberculosis (TB) bacilli: yes or no to preventive chemotherapy. Int J Mycobacteriol 2012;1:1-2.

2. Hibah NA, Hasan HE. Prevalence of latent tuberculosis infection among multinational healthcare workers in Muhayil Saudi Arabia. Egypt J Bronchol 2015;9:183-7.

3. Houben RM, Dodd PJ. The global burden of latent tuberculosis infection: a re-estimation using mathematical modelling. PLoS Med 2016;13:e1002152.

4. World Health Organization. Latent TB infection: updated and consolidated guidelines for programmatic management. Geneva: World Health Organization; 2018.

5. Balkhy HH, El Beltagy K, El-Saed A, Aljasir B, Althaqafi A, Alothman AF, et al. Comparison of QuantiFERON-TB gold in tube test versus tuberculin skin test for screening of latent tuberculosis infection in Saudi Arabia: a population-based study. Ann Thorac Med 2016;11:197-201.
6. Daniel TM. The history of tuberculosis. Respir Med 2006;100:1862-70.

7. Grosset J. The sterilizing value of rifampicin and pyrazinamide in experimental short-course chemotherapy. Bull Int Union Tuberc 1978;53:5-12.

8. Wayne LG. Dormancy of Mycobacterium tuberculosis and latency of disease. Eur J Clin Microbiol Infect Dis 1994;13:908-14.

9. Agarwal R. Treatment of latent tuberculous infection in India: is it worth the salt? Lung India 2005;22:105-6.

10. Huaman MA, Ticona E, Miranda G, Kryscio RJ, Mugruza R, Aranda E, et al. The relationship between latent tuberculosis infection and acute myocardial infarction. Clin Infect Dis 2018;66:886-92.

11. Velayati AA, Abeel T, Shea T, Konstantinovich Zhavnerko G, Birren B, Cassell GH, et al. Populations of latent Mycobacterium tuberculosis lack a cell wall: Isolation, visualization, and whole-genome characterization. Int J Mycobacteriol 2016;5:66-73.

12. McDermott W. Inapparent infection: relation of latent and dormant infections to microbial persistence. Public Health Rep 1959;74:485-99.

13. Adeiza MA. Diagnosis of latent tuberculosis infection: the tuberculin skin test and interferon gamma release assays. Ann Niger Med 201 1;5:35-7.

14. Zondervan NA, van Dam JC, Schaap PJ, Martins Dos Santos VA, Suarez-Diez M. Regulation of three virulence strategies of Mycobacterium tuberculosis: a success story. Int J Mol Sci 2018;19:347.

15. Welin A. Survival strategies of Mycobacterium tuberculosis inside the human macrophage. Linköping: Linköping University; 2011.

16. Curcic R, Dhandayuthapani S, Deretic V. Gene expression in mycobacteria: transcriptional fusions based on xylE and analysis of the promoter region of the response regulator mtrA from Mycobacterium tuberculosis. Mol Microbiol 1994;13:1057-64.

17. O'Neill MB, Mortimer TD, Pepperell CS. Diversity of $\mathrm{Myco-}$ bacterium tuberculosis across Evolutionary Scales. PLoS Pathog 2015;11:e1005257.

18. Beste DJ, Laing E, Bonde B, Avignone-Rossa C, Bushell ME, McFadden JJ. Transcriptomic analysis identifies growth rate modulation as a component of the adaptation of mycobacteria to survival inside the macrophage. J Bacteriol 2007;189:3969-76.

19. Voskuil MI, Visconti KC, Schoolnik GK. Mycobacterium tuberculosis gene expression during adaptation to stationary phase and low-oxygen dormancy. Tuberculosis (Edinb) 2004;84:218-27.

20. Lancaster CR, Kroger A. Succinate: quinone oxidoreductases: new insights from X-ray crystal structures. Biochim Biophys Acta 2000;1459:422-31.

21. Kana BD, Weinstein EA, Avarbock D, Dawes SS, Rubin H, 
Mizrahi V. Characterization of the cydAB-encoded cytochrome bd oxidase from Mycobacterium smegmatis. J Bacteriol 2001;183:7076-86.

22. Cole ST, Brosch R, Parkhill J, Garnier T, Churcher C, Harris D, et al. Deciphering the biology of Mycobacterium tuberculosis from the complete genome sequence. Nature 1998;393:537-44.

23. Pham TV, Murkin AS, Moynihan MM, Harris L, Tyler PC, Shetty N, et al. Mechanism-based inactivator of isocitrate lyases 1 and 2 from Mycobacterium tuberculosis. Proc Natl Acad Sci U S A 2017;114:7617-22.

24. Neidhardt FC, Curtiss R. Escherichia coli and Salmonella: cellular and molecular biology. Washington, DC: ASM Press; 1996. p. 1227-31.

25. Kumar R, Bhakuni V. Mycobacterium tuberculosis isocitrate lyase (MtbIcl): role of divalent cations in modulation of functional and structural properties. Proteins 2008;72:892900.

26. Smith CV, Huang CC, Miczak A, Russell DG, Sacchettini JC, Honer zu Bentrup K. Biochemical and structural studies of malate synthase from Mycobacterium tuberculosis. J Biol Chem 2003;278:1735-43.

27. Gengenbacher M, Kaufmann SH. Mycobacterium tuberculosis: success through dormancy. FEMS Microbiol Rev 2012;36:514-32.

28. Ellenbarger JF, Krieger IV, Huang HL, Gomez-Coca S, Ioerger TR, Sacchettini JC, et al. Anion-pi interactions in computer-aided drug design: modeling the inhibition of malate synthase by phenyl-diketo acids. J Chem Inf Model 2018;58:2085-91.

29. Wayne LG, Sohaskey CD. Nonreplicating persistence of mycobacterium tuberculosis. Annu Rev Microbiol 2001;55:13963.

30. Huang HL, Krieger IV, Parai MK, Gawandi VB, Sacchettini JC. Mycobacterium tuberculosis malate synthase structures with fragments reveal a portal for substrate/product exchange. J Biol Chem 2016;291:27421-32.

31. Kelkar DS, Kumar D, Kumar P, Balakrishnan L, Muthusamy B, Yadav AK, et al. Proteogenomic analysis of Mycobacterium tuberculosis by high resolution mass spectrometry. Mol Cell Proteomics 2011;10:M111.011627.

32. Berney M, Cook GM. Unique flexibility in energy metabolism allows mycobacteria to combat starvation and hypoxia. PLoS One 2010;5:e8614.

33. Parrish NM, Dick JD, Bishai WR. Mechanisms of latency in Mycobacterium tuberculosis. Trends Microbiol 1998;6:10712.

34. Wayne LG, Hayes LG. Nitrate reduction as a marker for hypoxic shiftdown of Mycobacterium tuberculosis. Tuber Lung Dis 1998;79:127-32.

35. Sohaskey CD, Wayne LG. Role of narK2X and narGHJI in hypoxic upregulation of nitrate reduction by Mycobacterium tuberculosis. J Bacteriol 2003;185:7247-56.
36. Sohaskey CD. Nitrate enhances the survival of Mycobacterium tuberculosis during inhibition of respiration. J Bacteriol 2008;190:2981-6.

37. Sohaskey CD. Regulation of nitrate reductase activity in Mycobacterium tuberculosis by oxygen and nitric oxide. Microbiology (Reading) 2005;151:3803-10.

38. Black GF, Thiel BA, Ota MO, Parida SK, Adegbola R, Boom $\mathrm{WH}$, et al. Immunogenicity of novel DosR regulon-encoded candidate antigens of Mycobacterium tuberculosis in three high-burden populations in Africa. Clin Vaccine Immunol 2009;16:1203-12.

39. Hutter B, Dick T. Analysis of the dormancy-inducible narK2 promoter in Mycobacterium bovis BCG. FEMS Microbiol Lett 2000;188:141-6.

40. Sohaskey CD, Modesti L. Differences in nitrate reduction between Mycobacterium tuberculosis and Mycobacterium bovis are due to differential expression of both narGHJI and narK2. FEMS Microbiol Lett 2009;290:129-34.

41. Honaker RW, Stewart A, Schittone S, Izzo A, Klein MR, Voskuil MI. Mycobacterium bovis BCG vaccine strains lack narK2 and narX induction and exhibit altered phenotypes during dormancy. Infect Immun 2008;76:2587-93.

42. Giffin MM, Raab RW, Morganstern M, Sohaskey CD. Mutational analysis of the respiratory nitrate transporter NarK2 of Mycobacterium tuberculosis. PLoS One 2012;7:e45459.

43. Kendall SL, Movahedzadeh F, Rison SC, Wernisch L, Parish T, Duncan K, et al. The Mycobacterium tuberculosis dosRS two-component system is induced by multiple stresses. Tuberculosis (Edinb) 2004;84:247-55.

44. Hutter B, Dick T. Up-regulation of narX, encoding a putative 'fused nitrate reductase' in anaerobic dormant Mycobacterium bovis BCG. FEMS Microbiol Lett 1999;178:63-9.

45. Fenhalls G, Stevens L, Moses L, Bezuidenhout J, Betts JC, van Helden P, et al. In situ detection of Mycobacterium tuberculosis transcripts in human lung granulomas reveals differential gene expression in necrotic lesions. Infect Immun 2002;70:6330-8.

46. Queiroz A, Riley LW. Bacterial immunostat: Mycobacterium tuberculosis lipids and their role in the host immune response. Rev Soc Bras Med Trop 2017;50:9-18.

47. Bekierkunst A. Acute granulomatous response produced in mice by trehalose-6,6-dimycolate. J Bacteriol 1968;96:95861.

48. Ishikawa E, Ishikawa T, Morita YS, Toyonaga K, Yamada H, Takeuchi O, et al. Direct recognition of the mycobacterial glycolipid, trehalose dimycolate, by C-type lectin Mincle. J Exp Med 2009;206:2879-88.

49. Rao V, Fujiwara N, Porcelli SA, Glickman MS. Mycobacterium tuberculosis controls host innate immune activation through cyclopropane modification of a glycolipid effector molecule. J Exp Med 2005;201:535-43.

50. Huang CC, Smith CV, Glickman MS, Jacobs WR Jr, Sacchettini JC. Crystal structures of mycolic acid cyclopropane 
synthases from Mycobacterium tuberculosis. J Biol Chem 2002;277:11559-69.

51. PupMed. pcaA: cyclopropane mycolic acid synthase (Mycobacterium tuberculosis H37Rv). Bethesda: National Library of Medicine; 2018.

52. Corrales RM, Molle V, Leiba J, Mourey L, de Chastellier C, Kremer L. Phosphorylation of mycobacterial PcaA inhibits mycolic acid cyclopropanation: consequences for intracellular survival and for phagosome maturation block. J Biol Chem 2012;287:26187-99.

53. Nishimoto T, Nakano M, Nakada T, Chaen H, Fukuda S, Sugimoto T, et al. Purification and properties of a novel enzyme, trehalose synthase, from Pimelobacter sp. R48. Biosci Biotechnol Biochem 1996;60:640-4.

54. Caner S, Nguyen N, Aguda A, Zhang R, Pan YT, Withers SG, et al. The structure of the Mycobacterium smegmatis trehalose synthase reveals an unusual active site configuration and acarbose-binding mode. Glycobiology 2013;23:107583.

55. Umesiri FE, Sanki AK, Boucau J, Ronning DR, Sucheck SJ. Recent advances toward the inhibition of $\mathrm{mAG}$ and LAM synthesis in Mycobacterium tuberculosis. Med Res Rev 2010;30:290-326.

56. Thanna S, Sucheck SJ. Targeting the trehalose utilization pathways of Mycobacterium tuberculosis. Medchemcomm 2016;7:69-85.

57. Zahrt TC, Deretic V. Mycobacterium tuberculosis signal transduction system required for persistent infections. Proc Natl Acad Sci U S A 2001;98:12706-11.

58. Marszalek M, Planas A, Pellicer T. Two-component systems of Mycobacterium tuberculosis as potential targets for drug development. Afinidad 2014;71:172-8.

59. Banerjee SK, Kumar M, Alokam R, Sharma AK, Chatterjee A, Kumar R, et al. Targeting multiple response regulators of Mycobacterium tuberculosis augments the host immune response to infection. Sci Rep 2016;6:25851.

60. Jordan S, Hutchings MI, Mascher T. Cell envelope stress response in Gram-positive bacteria. FEMS Microbiol Rev 2008;32:107-46.

61. Zhou P, Long Q, Zhou Y, Wang H, Xie J. Mycobacterium tuberculosis two-component systems and implications in novel vaccines and drugs. Crit Rev Eukaryot Gene Expr 2012;22:37-52.

62. Tyagi JS, Sharma D. Signal transduction systems of mycobacteria with special reference to $M$. tuberculosis. Curr Sci 2004;86:93-102.

63. Wisedchaisri G, Wu M, Sherman DR, Hol WG. Crystal structures of the response regulator DosR from Mycobacterium tuberculosis suggest a helix rearrangement mechanism for phosphorylation activation. J Mol Biol 2008;378:227-42.

64. Park HD, Guinn KM, Harrell MI, Liao R, Voskuil MI, Tompa $\mathrm{M}$, et al. Rv3133c/dosR is a transcription factor that mediates the hypoxic response of Mycobacterium tuberculosis.
Mol Microbiol 2003;48:833-43.

65. Sherman DR, Voskuil M, Schnappinger D, Liao R, Harrell MI, Schoolnik GK. Regulation of the Mycobacterium tuberculosis hypoxic response gene encoding alpha-crystallin. Proc Natl Acad Sci U S A 2001;98:7534-9.

66. Magombedze G, Dowdy D, Mulder N. Latent tuberculosis: models, computational efforts and the pathogen's regulatory mechanisms during dormancy. Front Bioeng Biotechnol 2013;1:4.

67. Kalscheuer R. Genetics of wax ester and triacylglycerol biosynthesis in bacteria. In: Timmis KN, editor. Handbook of hydrocarbon and lipid microbiology. Berlin: Springer; 2010. p. 527-35.

68. Kwon KW, Kim WS, Kim H, Han SJ, Hahn MY, Lee JS, et al. Novel vaccine potential of Rv3131, a DosR regulon-encoded putative nitroreductase, against hyper-virulent Mycobacterium tuberculosis strain K. Sci Rep 2017;7:44151.

69. Sharma S, Tyagi JS. Mycobacterium tuberculosis DevR/ DosR dormancy regulator activation mechanism: dispensability of phosphorylation, cooperativity and essentiality of alpha10 helix. PLoS One 2016;11:e0160723.

70. Dasgupta N, Kapur V, Singh KK, Das TK, Sachdeva S, Jyothisri K, et al. Characterization of a two-component system, devR-devS, of Mycobacterium tuberculosis. Tuber Lung Dis 2000;80:141-59.

71. Saini DK, Malhotra V, Dey D, Pant N, Das TK, Tyagi JS. DevR-DevS is a bona fide two-component system of $\mathrm{My}$ cobacterium tuberculosis that is hypoxia-responsive in the absence of the DNA-binding domain of DevR. Microbiology (Reading) 2004;150:865-75.

72. Perez E, Samper S, Bordas Y, Guilhot C, Gicquel B, Martin C. An essential role for phoP in Mycobacterium tuberculosis virulence. Mol Microbiol 2001;41:179-87.

73. Hutter B, Dick T. Increased alanine dehydrogenase activity during dormancy in Mycobacterium smegmatis. FEMS Microbiol Lett 1998;167:7-11.

74. Gonzalo-Asensio J, Mostowy S, Harders-Westerveen J, Huygen K, Hernandez-Pando R, Thole J, et al. PhoP: a missing piece in the intricate puzzle of Mycobacterium tuberculosis virulence. PLoS One 2008;3:e3496.

75. Bretl DJ, He H, Demetriadou C, White MJ, Penoske RM, Salzman NH, et al. MprA and DosR coregulate a Mycobacterium tuberculosis virulence operon encoding Rv1813c and Rv1812c. Infect Immun 2012;80:3018-33.

76. Pang X, Samten B, Cao G, Wang X, Tvinnereim AR, Chen $\mathrm{XL}$, et al. MprAB regulates the espA operon in Mycobacterium tuberculosis and modulates ESX-1 function and host cytokine response. J Bacteriol 2013;195:66-75.

77. Sachdeva P, Misra R, Tyagi AK, Singh Y. The sigma factors of Mycobacterium tuberculosis: regulation of the regulators. FEBS J 2010;277:605-26.

78. He H, Hovey R, Kane J, Singh V, Zahrt TC. MprAB is a stressresponsive two-component system that directly regulates 
expression of sigma factors SigB and SigE in Mycobacterium tuberculosis. J Bacteriol 2006;188:2134-43.

79. Agrawal R, Saini DK. Rv1027c-Rv1028c encode functional KdpDE two-component system in Mycobacterium tuberculosis. Biochem Biophys Res Commun 2014;446:1172-8.

80. Heermann R, Jung K. K+ supply, osmotic stress and the KdpD/KdpE two-component system. In: Gross R, Beier D, editors. Two-component systems in bacteria. Poole: Caister Academic Press; 2012. p. 181-99.

81. Haydel SE, Clark-Curtiss JE. Global expression analysis of two-component system regulator genes during Mycobacterium tuberculosis growth in human macrophages. FEMS Microbiol Lett 2004;236:341-7.

82. Folkvardsen DB, Norman A, Andersen AB, Rasmussen EM, Lillebaek T, Jelsbak L. A major Mycobacterium tuberculosis outbreak caused by one specific genotype in a low-incidence country: exploring gene profile virulence explanations. Sci Rep 2018;8:11869.

83. Parish T, Smith DA, Kendall S, Casali N, Bancroft GJ, Stoker NG. Deletion of two-component regulatory systems increases the virulence of Mycobacterium tuberculosis. Infect Immun 2003;71:1134-40.

84. Bhattacharya M, Biswas A, Das AK. Interaction analysis of TcrX/Y two component system from Mycobacterium tuberculosis. Biochimie 2010;92:263-72.

85. Haydel SE, Benjamin WH, Jr., Dunlap NE, Clark-Curtiss JE. Expression, autoregulation, and DNA binding properties of the Mycobacterium tuberculosis TrcR response regulator. J Bacteriol 2002;184:2192-203.

86. Haydel SE, Dunlap NE, Benjamin WH, Jr. In vitro evidence of two-component system phosphorylation between the Mycobacterium tuberculosis TrcR/TrcS proteins. Microb Pathog 1999;26:195-206.

87. Feklistov A, Sharon BD, Darst SA, Gross CA. Bacterial sigma factors: a historical, structural, and genomic perspective. Annu Rev Microbiol 2014;68:357-76.

88. Gruber TM, Gross CA. Multiple sigma subunits and the partitioning of bacterial transcription space. Annu Rev Microbiol 2003;57:441-66.

89. Rodrigue S, Provvedi R, Jacques PE, Gaudreau L, Manganelli R. The sigma factors of Mycobacterium tuberculosis. FEMS Microbiol Rev 2006;30:926-41.

90. Lonetto M, Gribskov M, Gross CA. The sigma 70 family: sequence conservation and evolutionary relationships. J
Bacteriol 1992;174:3843-9.

91. Beaucher J, Rodrigue S, Jacques PE, Smith I, Brzezinski R, Gaudreau L. Novel Mycobacterium tuberculosis anti-sigma factor antagonists control sigmaF activity by distinct mechanisms. Mol Microbiol 2002;45:1527-40.

92. Geiman DE, Kaushal D, Ko C, Tyagi S, Manabe YC, Schroeder BG, et al. Attenuation of late-stage disease in mice infected by the Mycobacterium tuberculosis mutant lacking the SigF alternate sigma factor and identification of SigFdependent genes by microarray analysis. Infect Immun 2004;72:1733-45.

93. Jeong EH, Son YM, Hah YS, Choi YJ, Lee KH, Song T, et al. RshA mimetic peptides inhibiting the transcription driven by a Mycobacterium tuberculosis sigma factor SigH. Biochem Biophys Res Commun 2006;339:392-8.

94. Li L, Fang C, Zhuang N, Wang T, Zhang Y. Structural basis for transcription initiation by bacterial ECF sigma factors. Nat Commun 2019;10:1153.

95. Fang C, Li L, Shen L, Shi J, Wang S, Feng Y, et al. Structures and mechanism of transcription initiation by bacterial ECF factors. Nucleic Acids Res 2019;47:7094-104.

96. Kaushal D, Schroeder BG, Tyagi S, Yoshimatsu T, Scott C, Ko $\mathrm{C}$, et al. Reduced immunopathology and mortality despite tissue persistence in a Mycobacterium tuberculosis mutant lacking alternative sigma factor, SigH. Proc Natl Acad Sci U S A 2002;99:8330-5.

97. Graham JE, Clark-Curtiss JE. Identification of Mycobacterium tuberculosis RNAs synthesized in response to phagocytosis by human macrophages by selective capture of transcribed sequences (SCOTS). Proc Natl Acad Sci U S A 1999; 96:11554-9.

98. Hernandez Pando R, Aguilar LD, Smith I, Manganelli R. Immunogenicity and protection induced by a Mycobacterium tuberculosis sigE mutant in a BALB/c mouse model of progressive pulmonary tuberculosis. Infect Immun 2010;78: 3168-76.

99. Banaiee N, Jacobs WR Jr, Ernst JD. Regulation of Mycobacterium tuberculosis whiB3 in the mouse lung and macrophages. Infect Immun 2006;74:6449-57.

100. Singh A, Crossman DK, Mai D, Guidry L, Voskuil MI, Renfrow MB, et al. Mycobacterium tuberculosis WhiB3 maintains redox homeostasis by regulating virulence lipid anabolism to modulate macrophage response. PLoS Pathog 2009;5:e1000545. 